\title{
Efectos del plomo en la salud de la niñez
}

Thomas D Matte, MC, MPH.(1)

\begin{abstract}
MatteTD.
Efectos del plomo en la salud de la niñez Salud Publica Mex 2003;45 supl 2:S220-S224. El texto completo en inglés de este artículo está disponible en: http://www.insp.mx/salud/index.html
\end{abstract}

\section{Resumen}

El plomo es un elemento que no tiene ninguna función fisiológica conocida en los seres humanos, pero cuyos efectos adversos inciden sobre una diversidad de procesos bioquímicos esenciales. Existe evidencia considerable acerca de los efectos adver sos so bre la salud de los niños del plomo en niveles que son comunes a distintas poblaciones en to do el mundo. La intoxicación aguda por plomo, que ocasiona encefalopatía, a pesar de no ser frecuente, sí pone en riesgo la vida y requiere de un tratamiento agresivo y oportuno. Es necesario tenerla presente en el diagnóstico diferencial de toda enfermedad no explicada que incluya anemia, convulsiones, letar go, dolor abdo minal, o vó mito recurrente. Existe una gran cantidad de niños que padecen los efectos subclínicos crónicos debidos a la exposición de bajo nivel al plomo, y que incluyen un desarrollo cognitivo deficiente, trastornos en la conducta, ligera deficiencia en la agudeza auditiva, y talla reducida. La evidencia disponible indica que las únicas inter venciones efectivas para evitar la intoxicación de bajo nivel por plomo son aquellas que se aplican para controlar la exposición a este metal. El texto completo en inglés de este artículo está disponible en:http://ww w.insp.mx/ salud/index.html

Palabras clave: plomo; desarrollo infantil; control de riesgo

\author{
MatteTD. \\ Effects of lead exposure on children's health. \\ Salud Publica Mex 2003;45 suppl 2:S220-S224. \\ The English version of this paper \\ is available at: http://www.insp.mx/salud/index.html
}

\begin{abstract}
A bstract
Lead is an element that has no known physiologic function in humans but adversely affects a variety of fundamental biochemical processes. A large body of evidence shows adverse health effects of lead in children at levels common in populations around the world. Acute lead poisoning with encephalo pathy, though infrequent, is life-threatening, requiring timely and aggressive treatment. It should be considered in the differential diagnosis of any unexplained illness that includes anemia, seizures, lethargy, abdominal pain, or recurrent vomiting. Many more children are harmed by chronic, subclinical effects of low-level lead exposure that include impaired cognitive development, adverse effects on behavior, subtle impairment of hearing acuity, and reduced height. Available evidence indicates that the only effective interventions to prevent low level lead toxicity are those that control lead exposure.The English version of this paper is available at: http://www.insp.mx/salud/index.html
\end{abstract}

Key words: lead, develo pment, childhood, exposure control
E 1 plomo es un elemento que no tiene ninguna fun— ción fisiológica conocida en el organismo humano. Antes de que se comenzara a extraer plomo de las minas, la presencia de este metal en el ambiente era relativamente reducida, de tal manera que el nivel "normal" de plomo en el organismo de los hombres prehistóricos era de apenas tres órdenes de magnitud por debajo de las cargas de plomo encontradas en el organismo de adultos norteamericanos al principio de la década de $1990 .{ }^{1}$ Durante las últimas décadas se ha acumulado

(1) D ivisión RiesgosA mbientales y sus Efectos sobre la Salud, Centro N acional de Salud A mbiental, Centros para la D etección y C ontrol de Enfermedades, Estados Unidos de América.

Fecha de recibido: 1 de febrero de 2002 - Fecha de aceptado: 24 de julio de 2002

Solicitud de sobretiros: Dr.Thomas D Matte. CUES, N YAM, 1216 Fifth Avenue, N ueva York, N Y 10029

Correo electrónico: tmatte.@ nyam.org 
una cantidad considerable de evidencia epidemiológica que demuestra que los niveles comunes de exposición al plomo en la población mundial ocasionan efectos adversos en la salud. ${ }^{2}$ Como sucede con muchos tóxicos ambientales, los niños en edad temprana son más susceptibles que los adultos debido a que tienden a ingerir y a absorber más plomo, en relación con su talla, y tomando en cuenta, por otro lado, la velocidad del desarrollo cerebral a esa edad. ${ }^{3}$

En la mayoría de los niños intoxicados por plomo los impactos son subclínicos, esto es, no ocasionan signos o síntomas evidentes al efectuar una revisión clínica de rutina. Sin embargo, los estudios a fondo sobre poblaciones infantiles muestran que la capacidad cognitiva, la conducta y el crecimiento de esos niños se ven más afectados, en promedio, cuando se les compara con aquellos que han estado menos expuestos al plomo. Estos efectos del plomo sobre la salud se han replicado en diversas poblaciones en múltiples estudios, y los resultados de la mayoría de éstos en animales son congruentes con los llevados a cabo en humanos. La medición de los niveles de plomo en sangre (NPS) ha sido ampliamente validada como un indicador de riesgo para la salud, si bien la oportunidad y el grado de elevación de los niveles asociados con el daño varían según el efecto que interesa.

En la siguiente parte de este estudio se consideran los posibles mecanismos de la intoxicación por plomo, se describe la intoxicación aguda por éste y se resume la evidencia de su intoxicación subclínica, crónica, en niños.

\section{Mecanismos de la intoxicación por plomo}

El plomo trastorna fundamentalmente los procesos bioquímicos de, virtualmente, todas las células y los sistemas del organismo. Se une a las proteínas, particularmente a aquellas de los grupos del sulfhidrilo, de tal manera que puede alterar su estructura y su función, $\mathrm{o}$ bien competir con otros metales en los sitios de enlace. ${ }^{4}$ Puesto que el plomo es químicamente similar al calcio, interfiere con diversos procesos dependientes de éste. Activa la proteína $\mathrm{C}$ quinasa $(\mathrm{PCQ})^{5}$, que es una enzima dependiente del calcio vinculada con el crecimiento y la diferenciación celular, la conservación de la barrera hematoencefálica, y la potenciación a largo plazo que se sospecha tiene que ver con la memoria. ${ }^{6}$ De hecho, la proteína $\mathrm{C}$ quinasa tiene una mayor afinidad con el plomo que el calcio, ${ }^{7}$ y la interferencia dependiente de dosis del plomo con la PCQ carece de un umbral evidente.

La baja exposición a plomo también interfiere con la síntesis del grupo hem. Si bien éste causa anemia sólo cuando los NPS se elevan a cerca de los $50 \mu \mathrm{g} / \mathrm{dl}$ la re- ducción en la reserva de hem del organismo, por NPS más bajos, puede tener consecuencias que incluyen la disminución de la producción de la energía celular neural, niveles reducidos de la vitamina $\mathrm{D}-1.25(\mathrm{OH})_{2}$ y deficiencia de la acción metabólica hepática para medicamentos y tóxicos ambientales. ${ }^{8}$

\section{Intoxicación aguda por plomo en niños}

En New Hampshire, en abril de 2000, una niña de 30 meses de edad falleció a consecuencia de intoxicación por plomo; se trata del primer caso pediátrico de muerte por intoxicación por plomo reportado en una década. ${ }^{9}$ La presentación y el curso que siguió este caso ilustran algunos aspectos importantes de la intoxicación aguda por plomo en niños, así como las complicaciones potencialmente fatales de la encefalopatía por plomo. El 29 de marzo de 2000 esta niña fue llevada al servicio de urgencias de un hospital de primer nivel de atención, pues desde el día anterior presentaba fiebre baja y vómito. Los resultados de los análisis que se le practicaron indicaron la presencia de anemia microcítica con ocasional punteado basófilo de los eritrocitos. Se le administraron antibióticos para combatir una supuesta infección por estreptococos en la garganta, así como un antiemético. El 17 de abril fue llevada de nueva cuenta al servicio de urgencias del mismo hospital debido a que el vómito había empeorado, y fue hospitalizada. Al día siguiente fue transferida a un hospital de tercer nivel de atención donde se observó que presentaba un cierto estado letárgico. A las pocas horas de su ingreso al hospital la paciente súbitamente dejó de responder; presentaba apnea y estaba hipotensa. Fue transferida a un respirador artificial y se le administraron medicamentos vasopresores para mantener su presión arterial. Una tomografía mostró la presencia de edema cerebral difuso, con dilatación de los ventrículos. Por otra parte, los resultados de un examen de plomo en sangre practicados en una muestra tomada en días anteriores indicaron un nivel de 391 $\mu \mathrm{g} / \mathrm{dl}$. El nivel de protoporfirina eritrocítica era de $541 \mu \mathrm{g} / \mathrm{dl}$. La paciente siguió sin responder a pesar de habérsele administrado terapia de quelación y de que hubo un rápido descenso de los NPS. El 21 de abril se declaró la muerte cerebral.

Afortunadamente hoy en día la encefalopatía por plomo es rara en los Estados Unidos de América, pero tal cual lo ilustra este caso, la condición puede tornarse fatal rápidamente. Como ha quedado descrito en la literatura, los síntomas de la intoxicación por plomo pueden incluir anorexia, irritabilidad, disminución de la actividad lúdica, y alteración del patrón de sueño. Estos síntomas se pueden presentar a partir de los 
$50 \mu \mathrm{g} / \mathrm{dl}$ en adelante de plomo en sangre. Cuando aumentan los niveles, o bien, se prolonga la exposición a plomo, puede haber dolor abdominal y vómito. En el caso de la intoxicación crónica por plomo, en ocasiones aparecen las "líneas de plomo" en las encías, las líneas de detención del crecimiento que se pueden apreciar mediante la radiografía, y el punteado basófilo de los eritrocitos. Empero, estos signos no bastan para diagnosticar o determinar la intoxicación por plomo: es necesario detectar el NPS si se sospecha tal condición. ${ }^{10}$

Con una exposición continua, los síntomas pueden aumentar o disminuir, o bien, puede no haber síntomas previos a la súbita aparición de la encefalopatía, la misma que ocurre, en la mayoría de los casos notificados, cuando los NPS son de $100 \mu \mathrm{g} / \mathrm{dl}$ o más. Se ha asociado la encefalopatía por plomo con un aumento de la presión intercraneal, que se manifiesta por vómito, ataxia, estado fluctuante de la conciencia, coma y convulsiones.

La encefalopatía por plomo pone en riesgo la vida; es imperativo administrar un tratamiento oportuno y agresivo cuando existen NPS muy elevados. ${ }^{11}$ Debido a que los signos y síntomas de la intoxicación por plomo no son específicos, ésta deberá considerarse en todo diagnóstico diferencial de alguna enfermedad inexplicable, que incluya anemia, convulsiones, letargo, dolor abdominal, o bien vómito recurrente.

\section{Efectos de la baja exposición a plomo en el desarrollo neuronal}

La intoxicación subclínica por plomo debida a exposiciones "de bajo nivel" ha cobrado creciente importancia en el área de la salud pública, debido a la evidencia de que los NPS de tan sólo 10 a $20 \mu \mathrm{g} / \mathrm{dl}$ se han asociado con una disminución en el coeficiente intelectual (CI) promedio, de 2.5 puntos $^{12}$ y de un punto, ${ }^{13}$ respectivamente. A pesar de que una modificación de ese orden en el CI de un sujeto no resulta clínicamente evidente o claramente indicativa del desempeño futuro, sí tiene implicaciones desde el punto de vista de la salud pública, además de otras de carácter económico. Así por ejemplo, se ha estimado que un incremento de 10 $\mu \mathrm{g} / \mathrm{dl}$ de plomo en sangre, en un niño estadounidense, reduce en más de 10000 dólares $^{14}$ el valor actual de los ingresos económicos que percibirá en el futuro. Con la creciente participación de la tecnología en las economías mundiales es muy probable que también aumente el valor de la capacidad cognitiva en la fuerza laboral del futuro.

Sin embargo, hasta ahora no se ha identificado un umbral claro para esa relación dosis-efecto del plomo en sangre y el $\mathrm{CI}_{1}^{12}$ y parece que las deficiencias cogni- tivas debidas al plomo pueden persistir, al menos, hasta la adolescencia. ${ }^{15}$ La baja exposición a plomo también se ha asociado con efectos adversos en la conducta, incluyendo una disminución en la capacidad de concentración y un aumento en la impulsividad ${ }_{,}^{16}$ así como a una sutil deficiencia en la agudeza acústica, medida ésta con audiograma. ${ }^{17}$

La neuropatía periférica es una complicación, claramente descrita, de la intoxicación crónica por plomo. En el caso de los niños que, a pesar de tener NPS elevados no presentan ninguna neuropatía clínica, se ha relacionado de manera inversa la cantidad de plomo en sangre con la velocidad de la conducción nerviosa. $^{18}$

\section{Efectos del plomo sobre otros sistemas del organismo}

Además de la neurotoxicidad que afecta al desarrollo, también han quedado bastante bien descritos los efectos hematológicos del plomo. El metal interfiere en múltiples puntos del proceso de biosíntesis del grupo hem, incluyendo deficiencias en la hidratasa del ácido aminolevulínico (ALA-D) y la hierroquelasa. Este último mecanismo impide la incorporación del hierro a la protoporfirina hem precursora, que se acumula en el eritrocito en desarrollo. ${ }^{8}$ La medición de la protoporfirina eritrocítica $(\mathrm{PE})$-conocida como protoporfirina del zinc cuando se medía con un hematofluorómetro portátil-se utilizó alguna vez como medio para detectar la intoxicación por plomo. Sin embargo, puesto que es imposible detectar los niveles elevados de PE, atribuibles a la intoxicación por plomo, cuando los NPS son bajos, esto es, de 25 a $30 \mu \mathrm{g} / \mathrm{dl}$, esta prueba no es lo bastante sensible como para detectar NPS que hoy en día se consideran elevados. ${ }^{19}$

Cuando los NPS se elevan por primera vez, el PE se incrementa únicamente con la producción de eritrocitos. Así entonces, si el nivel de plomo en sangre es considerablemente elevado, un PE normal sugerirá un incremento reciente de plomo en sangre. El aumento de PE también puede deberse a una deficiencia de hierro. ${ }^{10} \mathrm{Si}$ bien los decrementos de los dependientes de dosis en hematocrito (dose-dependent decreases in hematocrit) pueden ocurrir cuando los NPS son muy reducidos $-20 \mu \mathrm{g} / \mathrm{dl}-$, es poco usual encontrar una franca anemia atribuible a la exposición a plomo en niveles de éste en sangre por debajo de los $40 \mu \mathrm{g} / \mathrm{dl} .^{20}$

En los niños gravemente intoxicados por plomo la intoxicación renal muy a menudo se presenta como una disfunción tubular proximal con glucosuria, aminoaciduria y fosfaturia ${ }^{21}$ manifiestas, conocida como sín- 
drome de Fanconi. A pesar de que generalmente se consideran reversibles estas manifestaciones agudas se notificó la persistencia de glucosuria y de aminoaciduria de 8 a 13 años después de haberse aplicado el tratamiento para la intoxicación pediátrica grave por plomo. ${ }^{22}$ Considerando que los NPS de los niños afectados eran en promedio de $18.5 \mu \mathrm{g} / \mathrm{dl}$-es decir, muy bajos para tomarse en cuenta como causa de una franca disfunción renal-, parecía tratarse más bien de la persistencia de una lesión tubular aguda. En un estudio de seguimiento de niños intoxicados por plomo en Queensland se notificó un exceso de muertes por nefritis e hipertensión, a menudo con hiperuricemia y gota coexistentes. ${ }^{23,24} \mathrm{La}$ evidencia de intoxicación renal crónica en niños sin antecedentes de intoxicación aguda por el metal es limitada, pero se ha notificado una relación directa de plomo en sangre con la excreción de proteína en urina. ${ }^{25}$

Los estudios en adultos arrojan una evidencia más amplia de intoxicación renal crónica por plomo. Es posible que en los adultos que están laboralmente expuestos, y que presentan elevados niveles de este metal en sangre por periodos prolongados, la tasa de filtración glomerular haya disminuido o bien que presenten franca azotemia. Los hallazgos patológicos en la intoxicación renal crónica por plomo no son específicos. La intoxicación crónica también se ha vinculado con la gota y la hipertensión. Los estudios de poblaciones de adultos que presentan niveles más bajos de plomo en sangre muestran una relación inversa de los NPS con la eliminación de creatinina. La excreción de ciertas proteínas tubulares aumenta ante niveles medianamente bajos de exposición a plomo. ${ }^{26}$

Los NPS se han asociado directamente con la presión arterial en adultos, inclusive cuando dichos niveles llegan a ser de $5 \mathrm{a} 10 \mu \mathrm{g} / \mathrm{dl} .^{27}$ No obstante, existe cierta incongruencia en los estudios respecto a si la presión sistólica o diástolica fue afectada fundamentalmente. ${ }^{26}$ Los estudios de seguimiento de largo plazo de niños intoxicados por plomo apoyan la posibilidad de un riesgo incrementado de hipertensión y enfermedad cardiovascular. Los estudios llevados a cabo en animales son congruentes con los datos obtenidos en humanos.

En los estudios transversales y longitudinales el peso de la evidencia indica la existencia de un ligero efecto adverso del plomo sobre la talla. ${ }^{28-30}$ En niños mexicano-norteamericanos de 5 a 12 años de edad que participaron en la Encuesta para Analizar la Nutrición y la Salud de la Población de Origen Hispánico -Hispanic Health and Nutrition Examination Survey (HANES, por sus siglas en inglés) la estatura de los niños que tenían NPS por encima de la mediana $(\sim 10$ $\mu \mathrm{g} / \mathrm{dl}$ ) era, en promedio, $1.2 \mathrm{~cm}$ inferior a la de los niños cuyos niveles estaban por debajo de la mediana. ${ }^{28}$

\section{Conclusión}

Existe abundante evidencia en humanos en cuanto a que la intoxicación por plomo, en particular la neurotoxicidad ambiental, ocurre en los niños cuando el plomo en sangre se encuentra en niveles que son bastante comunes en todo el mundo. Esta evidencia es el resultado de varios estudios llevados a cabo en diversas poblaciones y en los cuales se aplicaron diversos diseños, y está apoyada, a su vez, por evidencia procedente de estudios en animales. La intoxicación aguda por plomo puede presentarse con síntomas no específicos sutiles, o bien con ningún síntoma, antes de que ocurra un rápido deterioro. Se requiere de un elevado índice de sospecha, así como de la medición de los NPS, para poder efectuar un diagnóstico oportuno. No obstante, debido a la distribución log-normal del plomo en sangre, hay muchos más niños afectados por los efectos subclínicos de la exposición de bajo nivel a plomo, que por la intoxicación clínica de dicho metal. La evidencia disponible indica que las únicas intervenciones efectivas en la prevención de la intoxicación por plomo de bajo nivel son las mismas que se aplican para controlar la exposición a este metal.

\section{Referencias}

1. Patterson C, Ericson J, Manea-Krichten M, Shirahata H. N atural skeletal levels of lead in $\mathrm{H}$ omo sapiens sapiens uncontaminated by technological lead. Sci Total Environ 1991;107:205-236.

2.The $\mathrm{N}$ ational A cademy of Sciences $\mathrm{C}$ ommittee on Measuring Lead in Critical Populations. Measuring lead exposure in infants children and other critical populations. W ashington DC: N ational Academy Press, 1993.

3. Landrigan PJ, C arlson JE, Bearer C F, Cranmer JS, Bullard RD, Etzel $R A$ et al. Children's health and the environment: $A$ new agenda for prevention research. Environ Health Perspect 1998;106 Suppl 3: 787-794.

4. Goering PL. Lead-protein interactions as a basis for lead toxicity. N eurotoxicology 1993;14:45-60.

5. Markovac J, Goldstein GW. Lead activates protein kinase $C$ in immature rat brain microvessels. Toxicol A ppl Pharmacol 1988;96:14-23. 6. Suzuki T. Protein kinases involved in the expression of long-term potentiation. Int J Biochem 1994;26:735-744.

7. Markovac J, Goldstein GW. Picomolar concentrations of lead stimulate brain protein kinase C. N ature 1988;334:71-73.

8. US Environmental Protection A gency. Air quality criteria for lead. Research Triangle Park (NC): USEPA, 0 ffice of Research and Development, EPA 600/8-83-028F, 1986. 
9. US C enters for Disease Control and Prevention. Fatal pediatric lead poisoning-N ew Hamphshire, 2000. MMW R Morb Mortal W kly Rep 2001;50:457-459.

10. Center for Disease Control and Prevention. Preventing lead poisoning in young children: $A$ statement by the $C$ enters for $D$ isease Control, $O$ ctober 1991. Atlanta (GA): US D epartment of Health and Human Services, Public Health Service, CD C, 1991.

11. A merican A cademy 0 f Pediatrics Committee $0 \mathrm{n}$ D rugs. Treatment Guidelines for Lead Exposure in Children. Pediatrics 1995;96:155-160. 12. Schwartz J. Low-level lead exposure and children's IQ : A metaanalysis and search for a threshold. Environ Res 1994;65:42-55. 13. Pocock SJ, Smith M, Baghurst P. Environmental lead and children's intelligence:A systematic review of the epidemiological evidence. BMJ 1994;309:1189-1197.

14. Centers for Disease Control and Prevention. Strategic Plan for the Elimination of Childhood Lead Poisoning.Atlanta (GA) US D epartment of Health and Human Services, 1991.

15. N eedleman HL, Schell A, Bellinger D, Leviton A, Allred EN .The long-term effects of exposure to low doses of lead in childhood.An 11-year follow-up report. N Engl J Med 1990;322:83-88. 16. Rice DC. Behavioral effects of lead: Commonalities between experimental and epidemiologic data. Environ Health Perspect 1996;104 Suppl 2:337-351.

17. Schwartz J, 0 tto D. Blood lead, hearing thresholds, and neurobehavioral development in children and youth. Arch Environ Health 1987:42:153-160.

18. Landrigan PJ, Baker EL Jr, Feldman RG, Cox DH, Eden KV, O renstein W A. Increased lead absorption with anemia and slowed nerve conduction in children near a lead smelter. J Pediatr 1976;89:904-910. 19. McElvaine MD, 0 rbach HG, Binder S, Blanksma LA, Maes EF, Krieg RM. Evaluation of the erythrocyte protoporphyrin test as a screen for elevated blood lead levels. J Pediatr 1991;119:548-550.
20. Schwartz J, Landrigan PJ, Baker EL. Lead-induced anemia: D ose-response relationships and search for a threshold.Am J Public Health 1990;80: 165-168.

21. Chisholm JJ Jr, Harrison HC, Eberlien W R, Harrison HE.

A minoaciduria, hypophosphatemia, and rickets in lead poisoning: Study of a case. Am J D is Child 1955;89:159-168.

22. Loghman-Adham M.Aminoaciduria and glycosuria following severe childhood lead poisoning. Pediatr N ephrol 1998;12:218-221.

23. Henderson DA.A follow-up of cases of plumbism in children. Aust Ann Med 1954;3:219-224.

24. Inglis JA, Henderson DA, Emmerson BT.The pathology and pathogenesis of chronic lead nephropathy occurring in Q ueensland. J Pathol 1978;124:65-76.

25. Bernard AM, Vyskocil A, Roels H, Kriz J, Kodl M, Lauwerys R. Renal effects in children living in the vicinity of a lead smelter. Environ Res 1995;68:91-95.

26. Loghman-Adham M. Renal effects of environmental and occupational lead exposure. Environ Health Perspect 1997;105:928-939.

27. Schwartz J. Lead, blood pressure, and cardiovascular disease in men. Arch Environ Health 1995;50:31-37.

28. Frisancho AR, Ryan AS. Decreased stature associated with moderate blood lead concentrations in Mexican-A merican children. Am J Clin N utr 1991;54:516-519.

29. Ballew C, Khan LK, Kaufmann R, Mokdad A, Miller DT, Gunter EW. Blood lead concentration and children's anthropometric dimensions in the Third $\mathrm{N}$ ational Health and $\mathrm{N}$ utrition Examination Survey (N HAN ES III), 1988-1994.J Pediatr 1999;134:623-630.

30. Shukla R, Dietrich KN , Bornschein RL, Berger O, Hammond PB. Lead exposure and growth in the early preschool child:A follow-up report from the Cincinnati Lead Study. Pediatrics 1991;88:886-892. 